\title{
ESTIMATION OF ERRORS IN THE TRANSVERSE VELOCITY VECTORS DETERMINED FROM HINODE/SOT MAGNETOGRAMS USING THE NAVE TECHNIQUE
}

\author{
Jongchul ChaE ${ }^{1}$ and Yong-Jae MoOn $^{2}$ \\ ${ }^{1}$ Astronomy Program, Department of Physics and Astronomy, Seoul National University, Seoul 151-747, Korea \\ ${ }^{2}$ Department of Astronomy \& Space Science, Kyung Hee University, Yongin-shi, Kyungki-do 446-701, Korea \\ E-mail: moonyj@khu.ac.kr
}

(Received February 1, 2009; Accepted May 20, 2009)

\begin{abstract}
Transverse velocity vectors can be determined from a pair of images successively taken with a time interval using an optical flow technique. We have tested the performance of the new technique called NAVE (non-linear affine velocity estimator) recently implemented by Chae \& Sakurai using real image data taken by the Narrowband Filter Imager (NFI) of the Solar Optical Telescope (SOT) aboard the Hinode satellite. We have developed two methods of estimating the errors in the determination of velocity vectors, one resulting from the non-linear fitting $\sigma_{v}$ and the other $\epsilon_{u}$ resulting from the statistics of the determined velocity vectors. The real error is expected to be somewhere between $\sigma_{v}$ and $\epsilon_{u}$. We have investigated the dependence of the determined velocity vectors and their errors on the different parameters such as the critical speed for the subsonic filtering, the width of the localizing window, the time interval between two successive images, and the signal-to-noise ratio of the feature. With the choice of $v_{\text {crit }}=2$ pixel/step for the subsonic filtering, and the window FWHM of 16 pixels, and the time interval of one step (2 minutes), we find that the errors of velocity vectors determined using the NAVE range from around $0.04 \mathrm{pixel} / \mathrm{step}$ in high signal-to-noise ratio features $(S / N \sim 10)$, to $0.1 \mathrm{pixel} / \mathrm{step}$ in low signa-to-noise ratio features $(S / N \sim 3)$ with the mean of about $0.06 \mathrm{pixel} / \mathrm{step}$ where $1 \mathrm{pixel} / \mathrm{step}$ corresponds roughly to $1 \mathrm{~km} / \mathrm{s}$ in our case.
\end{abstract}

Key words : methods: data analysis — Sun: atmospheric motions - techniques: image processing

\section{INTRODUCTION}

With the development of high performance optical flow techniques and the advent of seeing-free solar images of sub-arc second resolution, it has been possible to determine transverse velocity vectors of moving features on the Sun better than was done previously. Using synthetic images, Chae \& Sakurai (2008) examined the performance of three different methods - the technique of local correlation tracking (LCT), the differential affine velocity estimator (DAVE), and the nonlinear affine velocity estimator (NAVE). As a result, they found that the NAVE is superior to the other methods in that they better detect subpixel motions, superpixels motions, and nonuniform motions.

Solar images produced by the Solar Optical Telescope (SOT, Tsuneta et al. 2008) aboard the Hinode satellite (Kosugi et al. 2007) are quite suitable for the determination of the transverse velocity field, for they are free from the troublesome seeing and have high angular resolution $\left(0.2^{\prime \prime}\right)$ and high cadence. Chae \& Sakurai (2008) and Chae et al. (2008a) applied NAVE to the magnetograms taken by the SOT using the Narrowband Filter Imager (NFI). The same method was also applied to investigate flows in a prominence using $\mathrm{H} \alpha$ images taken by the same telescope (Chae et al. 2008b). However, the probable errors in the determined velocity vectors have not been estimated.

We present how to estimate the probable errors in the velocity vectors determined from the NFI magnetograms using NAVE. We have found that the errors are sensitive to the data processing as well as the parameters of NAVE. Particularly important are the precise co-alignment of images and the suppression of the effect of solar oscillations on images.

\section{DATA PROCESSING}

\section{(a) Data}

We use Stokes $I$ and $V$ images of AR 10930 that were taken by the SOT using the NFI for about four hours from 19:08 to 23:58 UT of 2006 December 11 . The NFI was tuned to $-120 \mathrm{~m} \AA$ of the Fe $\mathrm{I} \lambda 6302.5$ line. The cadence of images was $120 \mathrm{~s}$, and each image has $2048 \times 1024$ pixels of $0.16^{\prime \prime}$ size. The first step of data processing is the subtraction of dark and bias from the images, which was done using the standard procedure (fg_dark_model.pro).

Corresponding Author: Y.-J. Moon 


\section{(b) Removal of Cosmic Ray Hits}

The removal of cosmic ray hits from images constitutes an important step of data processing since images taken in the space are often affected by such events. Our method consists of two steps: detection and correction. If the absolute value of the Laplacian of the logarithmic image values at a pixel is larger than a critical value defined by a prescribed size, the pixel and its nearest pixels are determined to be contaminated by a cosmic ray hit. This judgement is based on the belief that none of the features that are naturally imaged by the telescope can be sharper than the point spread function, and sharper features represent cosmic ray hits. The values at these pixels are replaced by the mean values of their neighborhoods. Since the neighboring pixels may also be contaminated by cosmic ray hits, the replacement procedure is iterated a few times.

\section{(c) Image alignment}

Stokes $I$ and $V$ images are spatially co-aligned in a few steps. First, the pointing direction of the telescope recorded in the headers of data files is used to transform every image with a given reference point as if this point is located at the center of the solar disk. This process tracks the reference point using the standard rate of solar rotation at the corresponding latitude. This first step is not perfect, however, so that the images may be misaligned one another by up to 10 pixels.

The second step is to align the images using the offset pixel values determined by maximizing the cross correlation of the $V$ image and a reference $V$ image. The image taken at the middle of the sequence is chosen as the reference image. The value of the maximum cross correlation and the accuracy of the alignment decrease with the time interval between an image and the reference image. The alignment error may be up to a few pixels. At this stage, we save the co-aligned $V / I$ images into a file.

The final step of alignment is carried out after the sub-region of interest is chosen and the subsonic filtering explained below is performed. We use the first sub-image of $V / I$ as the reference, and the second subimage is aligned to the reference using the technique of cross correlation. Then this image becomes the reference and the third image is aligned to this new reference, and so on. This final step leads to the finest alignment with sub-pixel accuracy.

\section{(d) Subsonic Filtering}

The subsonic filtering (Title et al. 1989) suppresses the power of the Fourier components in the spacetime that has a phase speed faster than a given value $v_{\text {crit }}$. We apply this filtering to sub-images representing regions of the field of view since the three-dimensional Fourier transform necessary for the filtering requires much computing time and lots of computer memory. For our purpose we selected one region of $512 \times 512$ pix- els representing sunspots.

The subsonic filtering is effective not only in reducing the effect of solar oscillations, but also in suppressing the noise. We have selected four values of critical values: $v_{\text {crit }}=1,2,3$ and infinite pixel/step. The last choice is nothing but no filtering. Generally speaking, the lower the critical speed is, the stronger the effect is.

\section{METHOD}

\section{(a) NAVE}

The principle of the affine velocity estimator (AVE) to be applied to solar magnetograms was presented by Schuck (2005). The linear version (differential affine velocity estimator, DAVE) was implemented by Schuck (2006) and the non-linear version (NAVE) by Chae \& Sakurai (2008). The comparison of LCT, DAVE, and NAVE was done by Chae \& Sakurai.

Here we suppose that $\boldsymbol{r} \equiv(x, y)$ represents one of the grids in a discrete kernel so that $x$ and $y$ should have integer values, while $\boldsymbol{R} \equiv(X, Y)$ does an arbitrary point on the plane so that $X$ and $Y$ can have floating values. For a given pair of successive images $F$ and $S$, the NAVE seeks the set of parameters $\boldsymbol{p} \equiv\left(U_{0}, V_{0}, U_{x}, U_{y}, V_{x}, V_{y}\right)$ that minimizes the merit function

$$
\chi^{2}(\boldsymbol{R} ; \boldsymbol{p}) \equiv \sum_{\boldsymbol{r} \in W}\left[\frac{g(\boldsymbol{r}, \boldsymbol{R} ; \boldsymbol{p})}{\sigma(\boldsymbol{r}, \boldsymbol{R})}\right]^{2} G(\boldsymbol{r})
$$

at a position $\boldsymbol{R}$ where

$$
\begin{gathered}
g(\boldsymbol{r}, \boldsymbol{R} ; \boldsymbol{p}) \equiv S\left(\boldsymbol{R}+\boldsymbol{r}+\frac{\boldsymbol{\xi}}{2}\right) e^{-\nu / 2}-F\left(\boldsymbol{R}+\boldsymbol{r}-\frac{\boldsymbol{\xi}}{2}\right) e^{+\nu / 2} \\
\boldsymbol{\xi} \equiv\left[\begin{array}{c}
U_{0} \\
V_{0}
\end{array}\right]+\left[\begin{array}{cc}
U_{x} & U_{y} \\
V_{x} & V_{y}
\end{array}\right]\left[\begin{array}{l}
x \\
y
\end{array}\right] \\
\nu \equiv-\left(U_{x}+V_{y}\right) .
\end{gathered}
$$

The parameter $\sigma(\boldsymbol{r}, \boldsymbol{R})$ represents the standard deviation of the probable error in $g(\boldsymbol{r}, \boldsymbol{R})$ resulting from the noises in the two images $F$ and $S$. For convenience, we suppose it is independent of the position. Then we have

$$
\sigma^{2}(\boldsymbol{r}, \boldsymbol{R})=\sigma_{F}^{2}+\sigma_{S}^{2}
$$

where $\sigma_{F}$ and $\sigma_{S}$ represent the standard deviations of noises in the two images, respectively.

The window function adopted for the present study

$$
G(\boldsymbol{r})=\exp \left[-\ln 2 \cdot(|\boldsymbol{r}| / h)^{2}\right]
$$

with the half width at the half maximum $h$. The local domain $W$ is a square satisfying $|x| \leq 2 h$ and $|y| \leq 2 h$.

The details of the minimization were given in Chae \& Sakurai (2008). The present work, however, uses the 


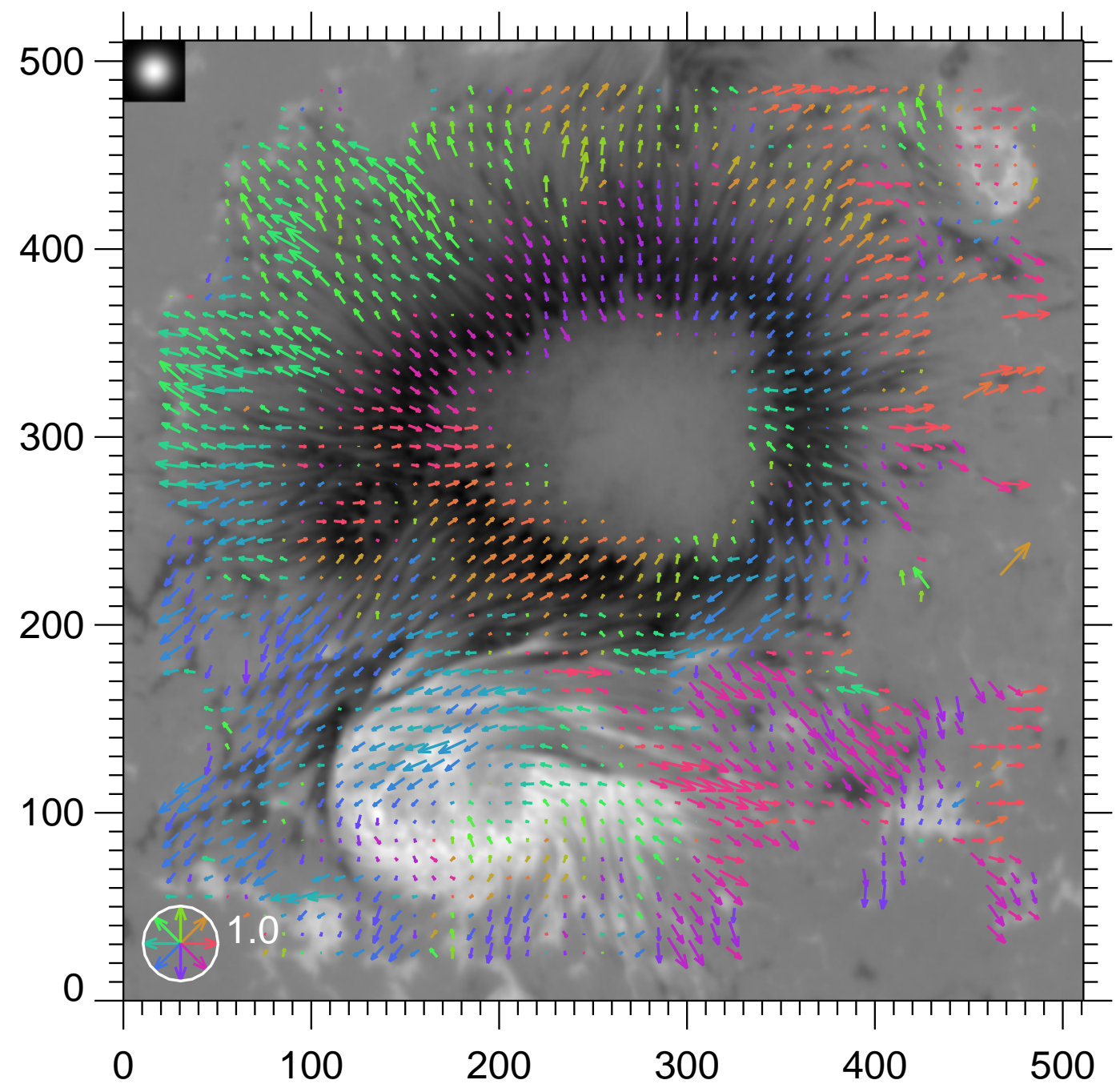

Fig. 1.- The spatial distribution of transverse velocity vectors. The used window function was inserted at the upper left corner. The arrows inside the circle in the lower left corner have the same magnitude (1 pxiel/step, or $0.97 \mathrm{~km} \mathrm{~s}^{-1}$ ), but have different directions expressed in different colors.

profile above while Chae \& Sakurai used a top-hat profile. Our experience indicates that the Gaussian profile produces the parameters that are spatially smoother than those produced with the use of the top-hat profile.

The major output of NAVE is, of course, the velocity vector

$$
\boldsymbol{V}=\left(\frac{U_{0}}{\Delta t}, \frac{V_{0}}{\Delta t}\right)
$$

defined at the position $\boldsymbol{R}$ and at the middle time between the two images $F$ and $S$. The parameter $\Delta t$ represents the time interval between the two images. For convenience, we measure $U_{0}$ and $V_{0}$ in the unit of pixel, and $\Delta t$ in the unit of time step, the smallest time interval that can be taken between two images in a sequence of images. In the present work, one pixel corresponds to $115 \mathrm{~km}$, one step to $120 \mathrm{~s}$, and hence one pixel/step to $0.97 \mathrm{~km} \mathrm{~s}^{-1}$

The standard errors of $\left(U_{0}, V_{0}, U_{x}, U_{y}, V_{x}, V_{y}\right)$ are given by $\sqrt{C_{k k}}(k=0,1,2, . ., 5)$ where $C_{k k}$ represents the $k$-th diagonal element of the inverse matrix of the Hesssian matrix

$$
\alpha_{l m}=\frac{1}{2} \frac{\partial^{2} \chi^{2}}{\partial p_{l} \partial p_{m}} .
$$

The standard error of velocity vector is then given by

$$
\sigma_{v}=\sqrt{C_{00}+C_{11}} / \Delta t .
$$

Note that this error is proportional to the noise in the data, so that it is important to provide a careful estimate of noise if $\sigma_{v}$ is to be estimated. Besides, $\sigma_{v}$ is inversely proportional to the number of data points in the domain $W$, so the larger the area for $W$ is the 

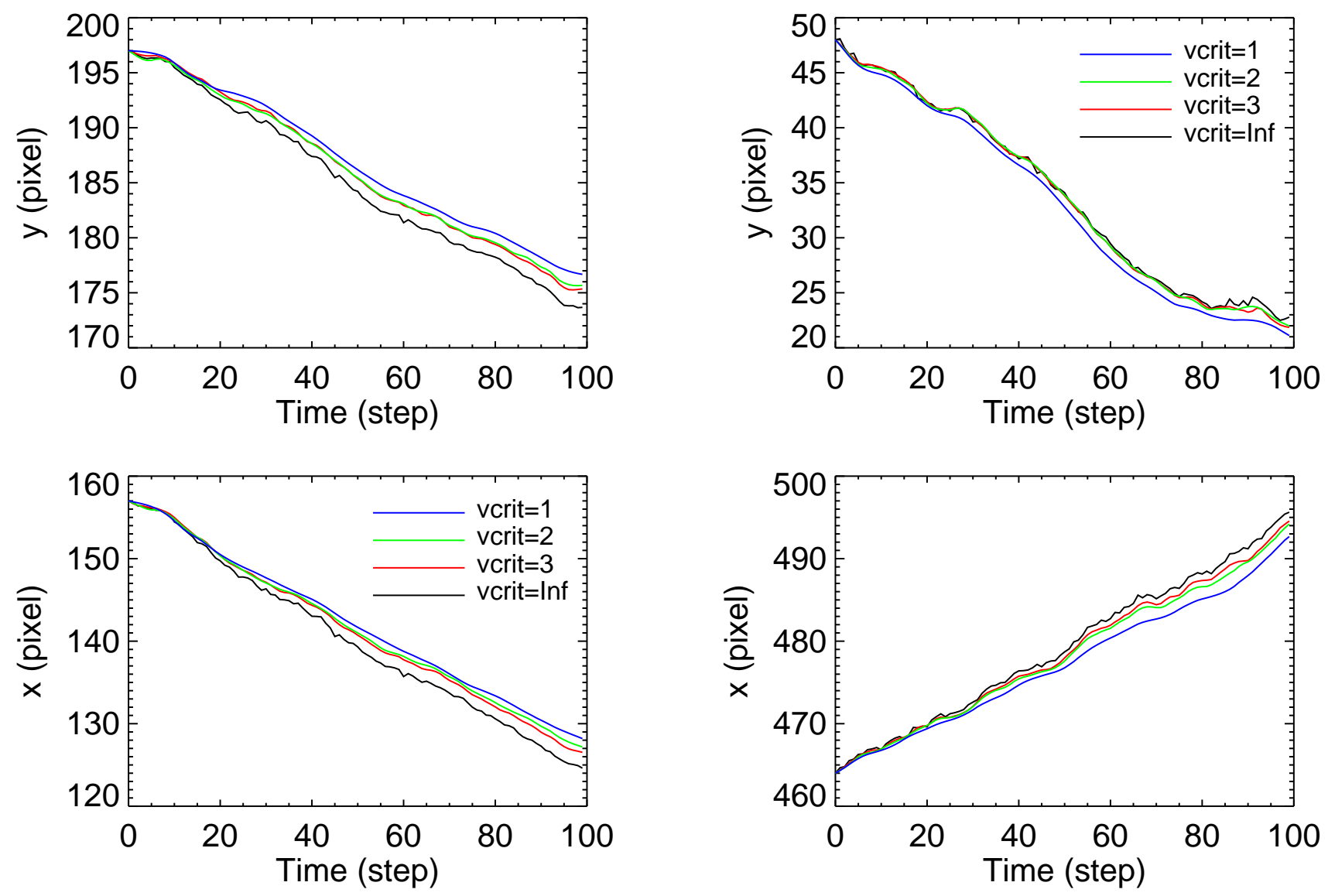

Fig. 2a.- Variations of $x$ and $y$ coordinates of a feature determined using raw data (critical speed is infinite) and subsonic-filtered data (critical speeds are 3,2 , and 1 pixel/step).

more precise the velocity measurement is as long as the velocity field varies little inside the chosen domain.

\section{(b) Tracking a Feature}

Suppose a feature is located at $\boldsymbol{R}_{k}$ at the $k$-th time step. We then ask where the feature goes in the $(k+1)$ th step. The solution is simple, being given by

$$
\boldsymbol{R}_{k+1} \equiv \boldsymbol{R}_{k}+\Delta \boldsymbol{R}_{k+1 / 2}
$$

provided the approximation

$$
\Delta \boldsymbol{R}_{k+1 / 2} \approx \boldsymbol{v}\left(\boldsymbol{R}_{k+1 / 2}, t_{k+1 / 2}\right)\left(t_{k+1}-t_{k}\right)
$$

is good enough with

$$
\begin{aligned}
\boldsymbol{R}_{k+1 / 2} & =\frac{\boldsymbol{R}_{k}+\boldsymbol{R}_{k+1}}{2} \\
t_{k+1 / 2} & =\frac{t_{k}+t_{k+1}}{2} .
\end{aligned}
$$



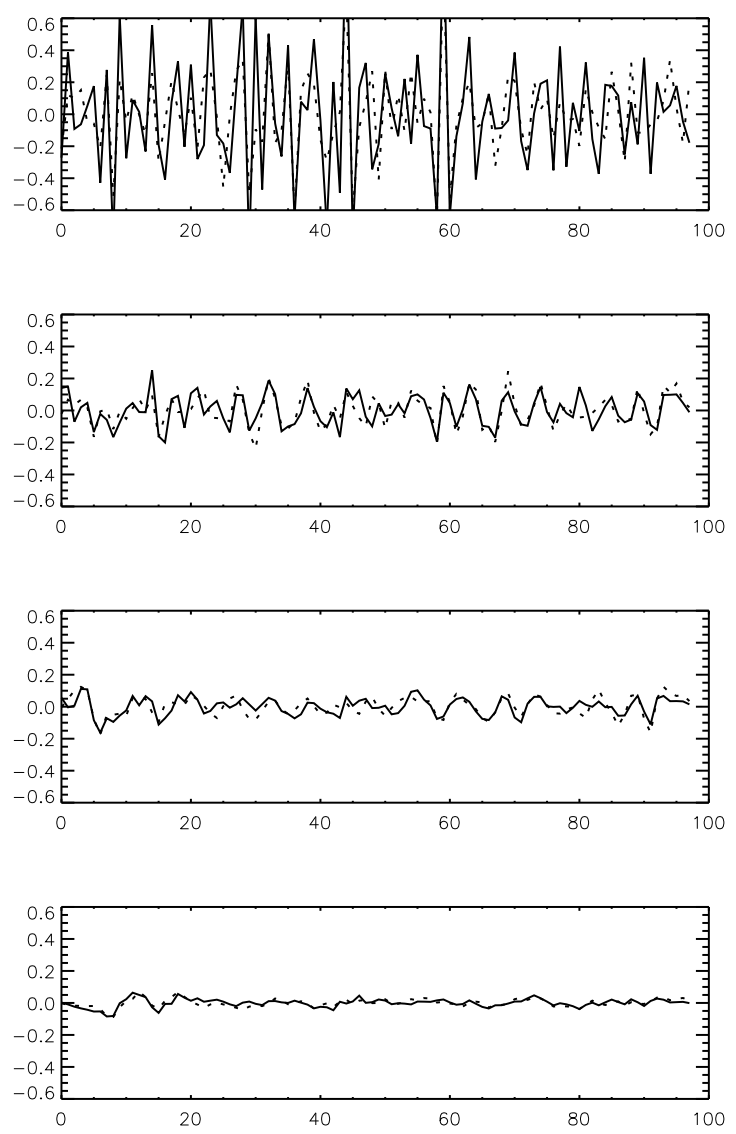

Fig. 3.- Variations of $\Delta V_{x}$ (solid) and $\Delta V_{y}$ (dotted) using unfiltered data and subsonic-filtered data with critical speeds of 3,2 , and 1 pixel /step from top to bottom.

is determined by the acceleration and the error superposition

$$
\Delta \boldsymbol{V}_{k}=\Delta t \boldsymbol{a}_{k}+\boldsymbol{n}_{k+1 / 2}-\boldsymbol{n}_{k-1 / 2}
$$

with $\Delta t \equiv\left(t_{k+1 / 2}-t_{k-1 / 2}\right)$. Suppose we have a number of values of $\Delta V_{k}(k=1, \ldots, N)$, and $\boldsymbol{a}_{k}, \boldsymbol{n}_{k+1 / 2}, \boldsymbol{n}_{k-1 / 2}$ are not correlated. Then it is possible to derive the relation

$$
\left\langle|\Delta \boldsymbol{V}-\langle\Delta \boldsymbol{V}\rangle|^{2}\right\rangle=\Delta t^{2}\left\langle|\boldsymbol{a}-\langle\boldsymbol{a}\rangle|^{2}\right\rangle+2\left\langle|\boldsymbol{n}|^{2}\right\rangle
$$

which gives the expression for an upper bound

$$
\epsilon_{u}=\left(\frac{1}{2}\left\langle|\Delta \boldsymbol{V}-\langle\Delta \boldsymbol{V}\rangle|^{2}\right\rangle\right)^{1 / 2}
$$

than which the error $\epsilon$ is not greater. Note the difference between $\epsilon$ and $\epsilon_{u}$ becomes small when the acceleration little varies with time.
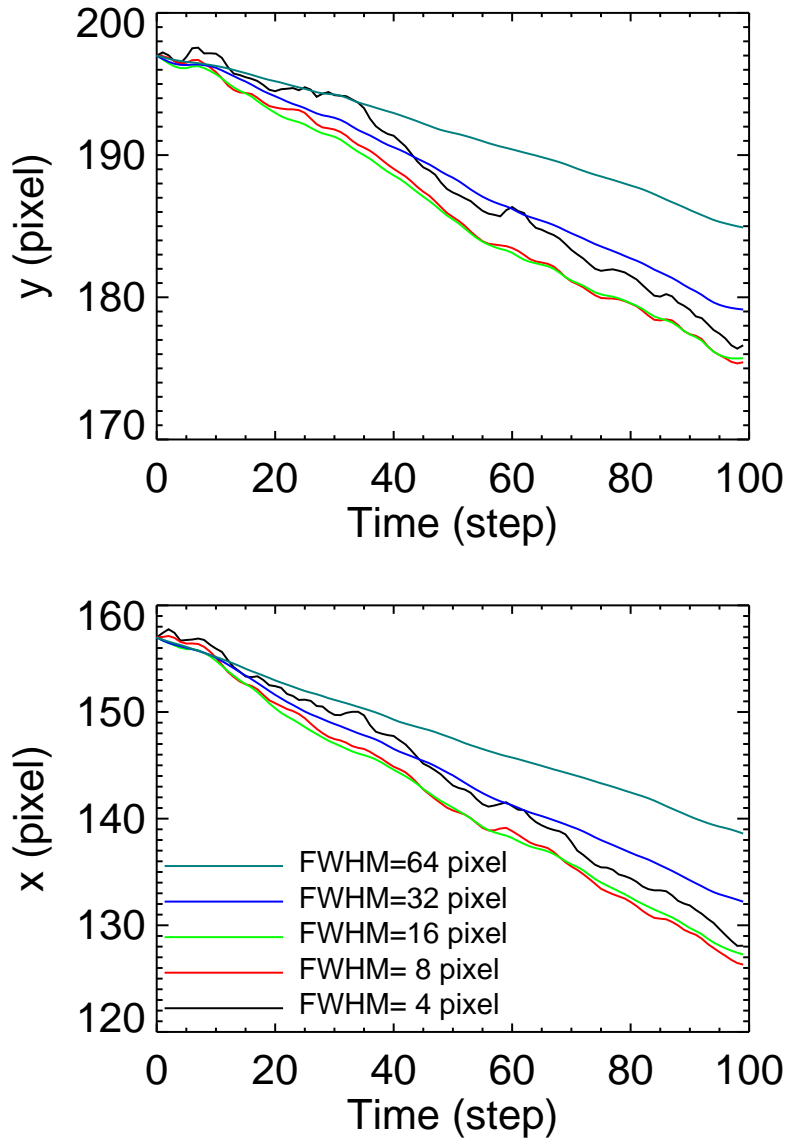

Fig. 4.- Variations of $x$ and $y$ coordinates of the feature determined using different widths of the window.

\section{RESULTS}

\section{(a) Velocity Maps}

Fig. 1 shows an example of a velocity map and its error map. Used are one image of $V / I$ taken at 20:08:41 UT of 2006 December 11 and the next image taken 2 minutes later. Subsonic-filtering with a critical speed of 2 pixel/step was applied to these images. The standard noise in the difference between these two images is estimated to be 0.0014 . Only the velocity vectors with standard errors smaller than $0.1 \mathrm{pixel} / \mathrm{step}$ are plotted in the map of velocity vectors in the left. The mean of the magnitudes of these vectors is $0.31 \mathrm{pixel} / \mathrm{step}$, and that of the corresponding errors is $0.059 \mathrm{pixel} / \mathrm{step}$.

\section{(b) Dependence on Subsonic Filtering}

Subsonic filtering strongly affects the velocity determination in a few ways. Fig. 2a shows several Lagrangian trajectories determined using different sets of data with different values of $v_{\text {crit }}$. They started from the same point, but their destinations are a bit differ- 


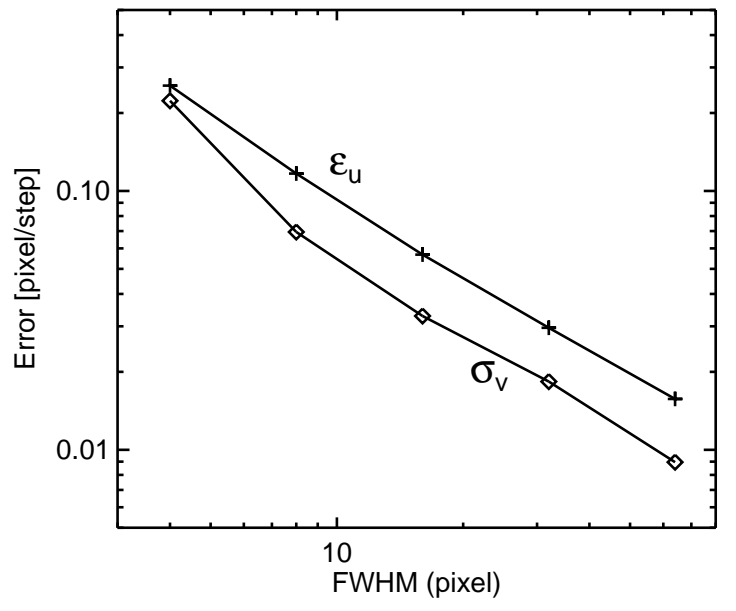

Fig. 5.- Dependence of the errors on the width of the window.

ent. The higher the $v_{\text {crit }}$ is, the farther away the destination is. The average velocity vector is $(-0.33,-0.24)$ $\mathrm{pixel} / \mathrm{step}$ in the case of raw data, and is $(-0.29,-0.21)$ pixel/step, for example, in the case $v_{\text {crit }}=1 \mathrm{pixel} / \mathrm{step}$. The ratio of this speed to that is 0.88 , suggesting the possibility that the subsonic filtering may have resulted in the underestimate of the average speed of a feature, even if it moves at a slower speed than $v_{\text {crit }}$. We have examined different starting points, and found that such a effect is weaker in other cases. As can be seen from Fig. 2b, the average speed is almost the same for the different values of $v_{\text {crit }}$ except for the case of $v_{\text {crit }}=1$ pixel/step. Therefore we think the systematic reduction of the average speed seen in Fig. 2a is not representative, but exceptional. This situation seems to occur when too strong subsonic filtering washes out some of faint real features. In this case, it may be necessary to have $v_{\text {crit }}$ high enough (e.g., $\geq 2 \mathrm{pixel} / \mathrm{step}$ ) to minimize such effect.

The advantage of subsonic filtering is well identified in Fig. 3 showing the variations of $\Delta \boldsymbol{V}$ (corresponding to the case in Fig. 2a). It is clear from the figure that the subsonic filtering reduces the amplitudes of the fluctuations in $\Delta \boldsymbol{V}$. The values of $\epsilon_{u}$ (defined in Eq. 18) are $0.328,0.098,0.058$, and $0.026 \mathrm{pixel} / \mathrm{step}$ for $v_{\text {crit }}=$ inf, $3,2,1 \mathrm{pixel} / \mathrm{step}$, respectively. These may be compared with the values of the standard error $\sigma_{v}$ (defined in Eq. 9): 0.050, 0.039, 0.033, $0.022 \mathrm{pixel} / \mathrm{step}$. In the case of raw data, $\epsilon_{u}$ is much larger than $\sigma_{v}$ while these are very close to each other in the case of $v_{\text {crit }}=1$ pixel/step. This contrast implies that the large amplitude fluctuation of velocity in the case of the raw data is mostly due to non-noisy signals which we think are artifacts in magnetograms that are induced by the solar oscillation. The application of subsonic filtering reduces, if not suppress, the systematic effects of the
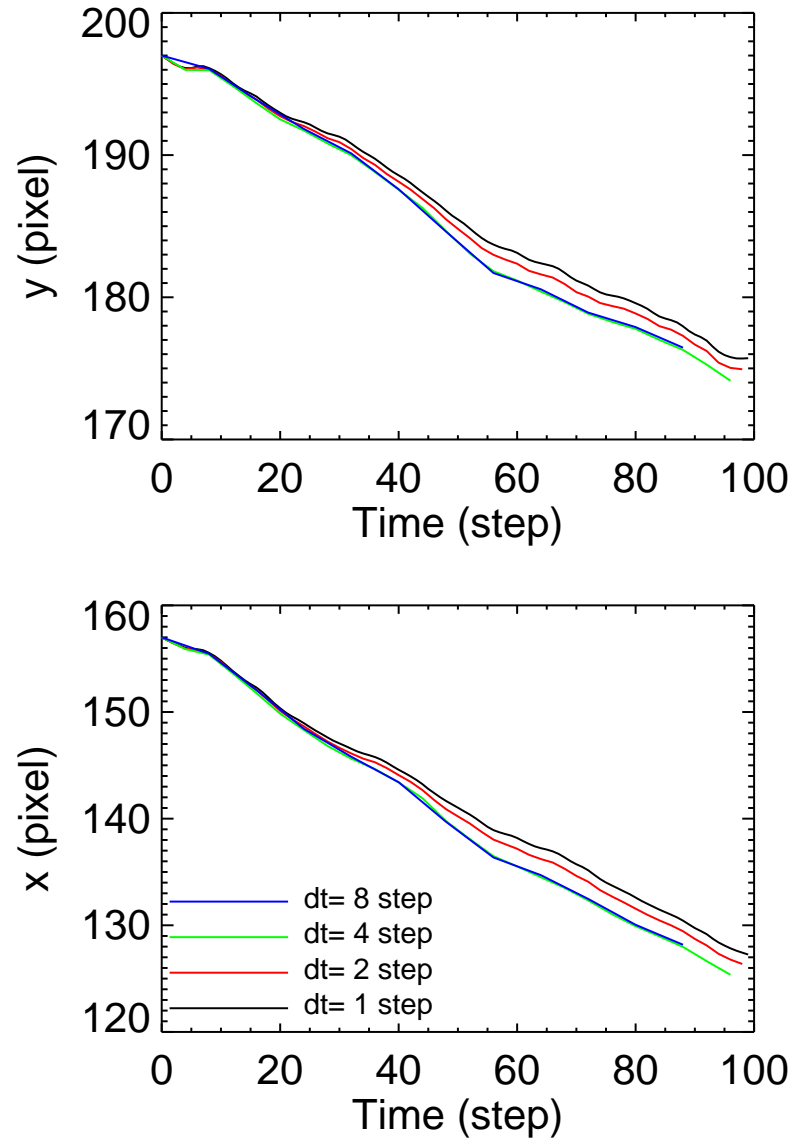

Fig. 6. - Variations of $x$ and $y$ coordinates of the feature determined using different time intervals.

solar oscillation as well as the random effect of noise. Note that the subsonic filtering, however, can not fully remove the systematic effect of solar oscillations. For example, the strong correlation between $\Delta V_{x}$ and $\Delta V_{y}$ (with the correlation coefficient of 0.72 in the case of $v_{\text {crit }}=2$ pixel/step ) indicates that the fluctuations are not due to the noise, but are mostly due to the oscillation of about $14 \mathrm{~min}$ period. We also find that the period of the highest power oscillation mode shifts to a longer value when a smaller value of $v_{\text {crit }}$ is adopted. For example, the dominant period of $\Delta \boldsymbol{V}$ fluctuation is $4.6 \mathrm{~min}$ in the raw data, and $7 \mathrm{~min}$ in the case of $v_{\text {crit }}=3 \mathrm{pixel} / \mathrm{step}$.

What would then be the optimal value of $v_{\text {crit }}$ among the four values? We choose $2 \mathrm{pixel} / \mathrm{step}\left(1.9 \mathrm{~km} \mathrm{~s}^{-1}\right)$ because it is above the speeds of most photospheric features and we think it can reduce the effect of oscillation reasonably well. With this choice, we find that the estimated error is smaller than $\epsilon_{u}=0.06 \mathrm{pixel} / \mathrm{step}$ in the case considered in Figs. $2 \& 3$. 


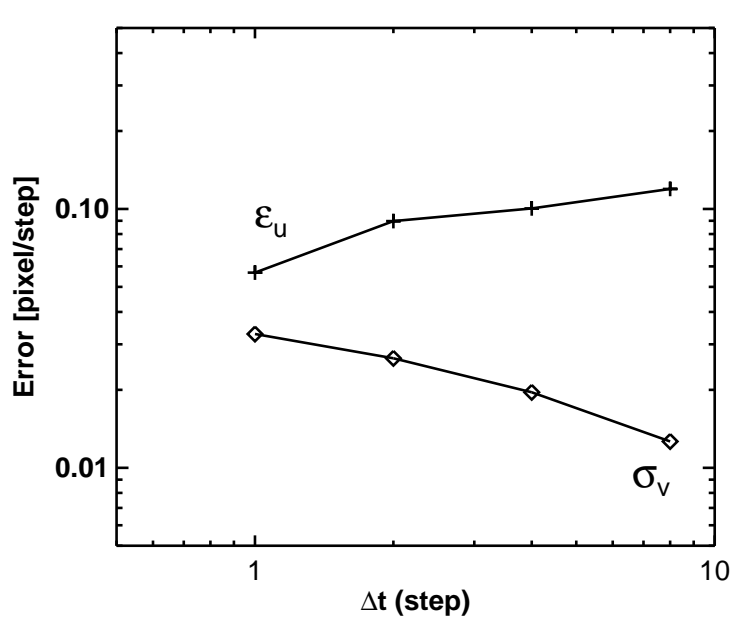

Fig. 7.- Dependence of the errors on the time interval.

\section{(c) Dependence on The Width of The Window}

We have tracked the same feature that in the previous section using the data subsonic filtered with 2 pixel/step and using different values of the width. It can be seen from Fig. 4 that if FHWM is too big (see the cases of 32 and 64 pixels), the speed may be systematically underestimated even though the trajectories look smooth. On the other hand, a too small value (see the case of 4 pixel) may yield a highly fluctuating trajectory which even may have a systematically underestimated average speed. Thus Fig. 4 suggests that either 8 or 16 pixels is better than the others.

Fig. 5 clearly shows that both the theoretical standard error $\sigma_{v}$ and the empirical error bound $\epsilon_{u}$ decrease with the width of the window. In the previous section, we already mentioned that $\epsilon_{u}$ is bigger than $\sigma_{v}$ because of the effect of solar oscillation. In the present case, the ratio of $\epsilon_{u}$ to $\sigma_{v}$ is about 1.7 . The real error may be between $\sigma_{v}$ and $\epsilon_{u}$. It can be seen from the figure that both $\sigma_{v}$ and $\epsilon_{u}$ tend to be inversely proportional to the FWHM of the window. This is consistent with our expectation that the errors are inversely proportional to the square root of the number of data points involved in the model fitting as can be inferred from Eqs. (1), $(8), \operatorname{and}(9)$.

\section{(d) Dependence on The Time Interval}

Fig. 6 shows that the trajectory of the feature is not sensitive to the time interval between two images. Note that the subsonic-filter data with $v_{\text {crit }}=2$ pixel/step were used and the FWHM of the window was set to 16 pixels. There is only a weak trend that the longer the time interval is, the higher the speed is, implying that the use of the longer time intervals is a little better for the tracking of the feature.

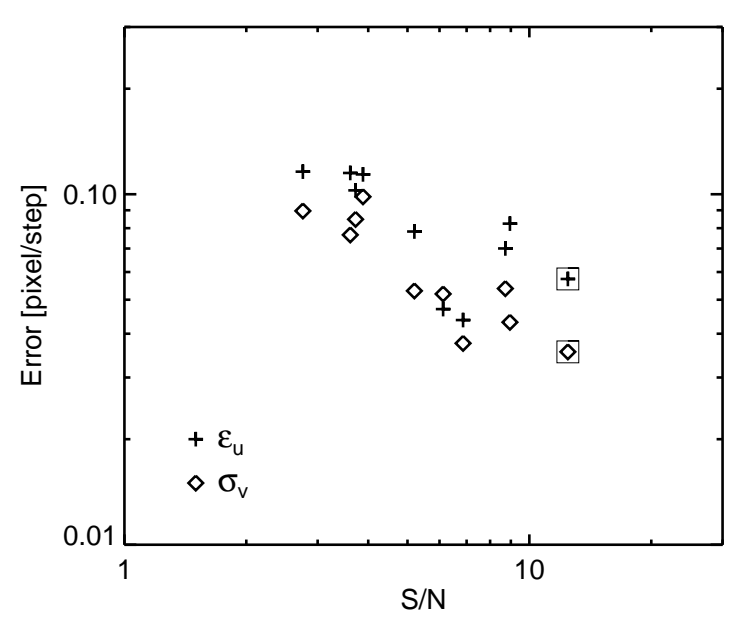

Fig. 8.- Dependence of the errors on the sginalto-noise ratio of the feature.

Fig. 7 shows the dependence of the errors on the time interval. We find that the variation of $\epsilon_{u}$ looks much different from that of $\sigma_{v}$. This is partly because $\epsilon_{u}$ increases with $\Delta t$ when $\Delta t$ becomes large enough because of the non-negligible effect of acceleration as can be seen from Eq. (17) while $\sigma_{v}$ is not subject to the effect of acceleration and decreases with $\Delta t$ as can be seen from Eq. (9). For this reason, we think that $\sigma_{v}$ may be closer to the real error than $\epsilon_{u}$, and that the error in the velocity decreases with $\Delta t$.

Let's understand how the time interval affects the measurement of the velocity of a feature. Provided that the feature does not change except for displacement, the NAVE technique can measure the super-pixel displacement - displacement bigger than one pixel - at the accuracy of an almost constant fraction of one pixel (Chae \& sakurai 2008). Therefore, the error in the velocity is inversely proportional to the time interval so that the longer time interval is preferred to the shorter one. The feature shown in Fig. 5 moves at a speed of about $0.4 \mathrm{pixel} / \mathrm{step}$. If the time interval of 8 step is chosen, the displacement during the time interval becomes about 3.2 pixel, which represents a super-pixel displacement. In principle, the error should be reduced by a factor of 8 . However, the reduction factor seen from the variation of $\sigma_{v}$ in Figure 7 is about 2.6, a value much smaller than 8 . The discrepancy may be attributed to the breakdown of the assumption that the feature does not change. During the time interval of 8 step (16 $\mathrm{min})$, the feature may have changed a lot, which may become another source of error in the determination of velocity. In reality, the time interval should be short enough to guarantee the near-constancy of a feature. 


\section{(e) Dependence on The Signal-to-Noise Ratio of The Feature}

The error in the measurement of velocity of a feature depends on its signal-to-noise ratio. It goes without saying that a conspicuous feature can be better tracked than a low-contrast feature for given noise. We define the signal as the standard deviation of a magnetogram inside a square region surrounding the center of the feature. The side of the square is set to the FWHM of the window. When a series of magnetograms are used, the average of the standard deviation is taken over the Lagrangian trajectory of the feature as a representative value of the feature.

Fig. 8 shows the values of $\sigma_{v}$ and $\epsilon_{u}$ versus the signal-to-noise ratios for ten selected features. We have used the subsonic filtered data with $v_{\text {crit }}=2 \mathrm{pixel} / \mathrm{step}$, and the time interval of one step, and the FWHM of the window of 16 pixels. The two enclosing squares indicate the feature we have investigated so far (see Figs. 2-7). We find that this feature has the highest signal-to-noise ration among the selected ones.

It is obvious from Fig. 8 that the error tends to decrease with the signal-to-noise ratio of the feature. The lowest-contrast features $(S / N \sim 3)$ have the errors about 0.1 , while the highest-contrast features $(S / N \sim 10)$ have the errors of about 0.04 pixel/step, with the errors being more or less inversely proportional to the signal-to-noise ratios.

\section{SUMMARY AND CONCLUSION}

We have tested the performance of the optical flow technique called non-linear affine velocity estimator (NAVE) using real data taken by the NFI of the SOT aboard the Hinode satellite. Specifically we have estimated the typical error in the velocity vectors determined using the NAVE. The present work complements our previous one (Chae \& Sakurai 2008) that was based on simulated data.

We have proposed two independent methods of error estimation. One is to use the inverse of the Hessian matrix. The standard error $\sigma_{v}$ that is obtained by this method is proportional to the noise in the data, so it is very important to estimate the noise. The other method is to use the statistics of velocity vectors obtained at different times while tracking a feature. The running differences of the velocity vectors are used to determine the upper bound of the standard deviation of the error $\epsilon_{u}$. Either if the time interval is short enough or if the feature moves at a constant velocity with a negligible acceleration, $\epsilon_{u}$ is a good proxy for the error. Usually $\epsilon_{u}$ is larger than $\sigma_{v}$, and the real error is expected to be somewhere between these two values.

Our results may be summarized as follows.

1. We confirmed that the subsonic filtering is effective in suppressing high-frequency fluctuations in the transverse velocity vectors. Solar oscillations affect the magnetograms taken by SOT/NFI so that velocity vectors determined from raw magnetograms have fluctuations as high as a few tenth of pixel/step, which is comparable to the speeds of true motion. The characteristic of the subsonic filtering depends on the critical speed chosen. The lower the critical speed is, the lower the amplitude and frequency of the fluctuation are. On the other hand, we find that the subsonic filtering may result in a systematic underestimation of the speed of a feature in some cases if the cut-off speed is too low. Therefore an optimal value of the critical speed is determined from a compromise between these two constraints. In the present study, the true speed appears to be lower than 1 pixel/step, and the value of $2 \mathrm{pixel} / \mathrm{step}$ may be an optimal choice for the critical speed.

2. The errors tend to be inversely proportional to the width of the localizing window. It should be kept in mind, however that the window width is taken to be too large, the speed of real motion may be seriously underestimated as well as the spatial resolution of the velocity field is degraded. In our case, a value in the range from 10 to 20 pixels appears to be an optimal choice for the FWHM of the window.

3. The errors also tend to be decrease with the time interval taken between two successive images, roughly being inversely proportional to the square root of it. A too short time interval may result in a slight underestimate of the speed while a too long time interval may be subject to serious errors because of the decrease of the cross-correlation between the two images as well as to the degradation of temporal resolution.

4. The errors tend to be inversely proportional to the signal-to-noise ratio of the feature. With the choice of $v_{\text {crit }}=2 \mathrm{pixel} / \mathrm{step}$, and the window FWHM of 16 pixels, and the time interval of 2 minutes, we find that the errors are around 0.04 pixel/step in high signal-to-noise ratio features $(S / N \sim 10)$, and around $0.1 \mathrm{pixel} / \mathrm{step}$ in low signa-to-noise ratio features $(S / N \sim 3)$.

The general conclusion we can draw from our results is that the optical flow technique we used best works when tractable features are clearly identified in a time series of images, which is not surprising at all. If there are no discernable features, there is no way to infer the velocity at all. Definitely images of higher signal-tonoise ratio with a large window will display more of such discernable features. Too strong subsonic filtering is not recommended since it may wash out some of faint real features.

\section{ACKNOWLEDGEMENTS}

This work was supported by the Korea Research Foundation Grant funded by the Korean Government 
(MOEHRD, Basic Research Promotion Fund) (KRF2008-314-C00158). The referee's comments are appreciated.

\section{REFERENCES}

Chae, J. \& Sakurai, T., 2008, A Test of Three Optical Flow Techniques-LCT, DAVE, and NAVE, ApJ, 689, 593

Chae, J., Litvinenko, Y. E., \& Sakurai, T., 2008a, Determination of Magnetic Diffusivity from High-Resolution Solar Magnetograms, ApJ, 683, 1153

Chae, J., Ahn, K., Lim, E.-K., Choe, G. S., \& Sakurai, T., 2008b, Persistent Horizontal Flows and Magnetic Support of Vertical Threads in a Quiescent Prominence, ApJL, 689, L73

Kosugi, T., et al., 2007, The Hinode (Solar-B) Mission: An Overview, Solar Phys., 243, 3

Schuck, P. W., 2005, Local Correlation Tracking and the Magnetic Induction Equation, ApJL, 632, L53

Schuck, P. W., 2006, Tracking Magnetic Footpoints with the Magnetic Induction Equation, ApJ, 646, 1358

Title, A., et al., 1989, Statistical properties of solar granulation derived from the SOUP instrument on Spacelab 2, ApJ, 336, 475

Tsuneta, S., et al., 2008, The Solar Optical Telescope for the Hinode Mission: An Overview, Solar Phys., 249, 167 